\title{
The adverse impact of malnutrition markers on major abdominopelvic cancer
} surgery

Niranjan J. Sathianathen ${ }^{1,2}$, Mary Kwaan ${ }^{3}$, Nathan Lawrentschuk ${ }^{1,4}$, Christopher J Weight $^{2}$, Simon P Kim ${ }^{5}$, Declan G Murphy ${ }^{4}$, Daniel A Moon ${ }^{4}$, Badrinath R. Konety ${ }^{2}$

1. University of Melbourne, Department of Surgery, Urology Unit and Olivia NewtonJohn Cancer Research Institute, Austin Health, Melbourne, Victoria, Australia

2. Department of Urology, University of Minnesota, Minneapolis, Minnesota, USA

3. Department of Surgery, University of Minnesota, Minneapolis, Minnesota, USA

4. Division of Cancer Surgery, Peter MacCallum Cancer Centre, Melbourne, Australia

5. Department of Urology, Case Western Reserve University, Cleveland, Ohio, USA

Corresponding author:

Dr Niranjan J Sathianathen

420 Delaware St SE,

MMC 394,

Department of Urology

University of Minnesota,

Minnesota, USA

E: nsathian@umn.edu

T: +1 660-334-0774

F: +16126260428

Abstract word count: 242

Text word Count: 2,424

References: 29

Tables: 1

Figures: 2

Supplementary appendices: 5

Conflicts of Interest: None

Sources of Funding: None

This is the author manuscript accepted for publication and has undergone full peer review but has not been through the copyediting, typesetting, pagination and proofreading process, which may lead to differences between this version and the Version of Record. Please cite this article as doi: 10.1111/ans.15129

This article is protected by copyright. All rights reserved. 


\section{Background}

Malnutrition has been associated with adverse postoperative outcomes in a range of procedures but none have evaluated the interaction between clinical indicators of malnutrition. We aimed to comparatively evaluate how combinations of nutritional parameters impact postoperative outcomes among patients undergoing major cancer operations.

\section{Methods}

Major abdominopelvic cancer surgery cases (colectomy, cystectomy, esophagectomy, gastrectomy, hysterectomy, nephrectomy, pancreatectomy, pneumonectomy and prostatectomy) were identified in the ACS-NSQIP database from 2007-2016. Malnutrition was defined by the presence of the following parameters: $\mathrm{BMI}<18.5 \mathrm{~kg} / \mathrm{m}^{2}$; preoperative serum albumin $<3.0 \mathrm{~g} / \mathrm{dL}$ or more than $10 \%$ weight loss in the last 6 months. Malnourished cases were matched with cases with satisfactory nutritional status using propensity scores. The primary outcome was the incidence of Clavien III-IV complications.

\section{Results}

Of the 30,207 cases included, $8.5 \%$ had at least one marker of malnutrition. The incidence of Clavien III-IV complications across all cases was $5.8 \%$. In the matched cohort, malnourished cases had a higher rate of complications than those with

This article is protected by copyright. All rights reserved. 
adequate nutritional status ( $11.3 \%$ vs $9.6 \%, p=0.018)$. A correlation was observed between the number of malnutrition markers possessed and the incidence of Clavien III-V complications. Cases with all three makers had the highest likelihood of experiencing a complication [OR 5.47, 95\% Cl 1.85-16.17].

\section{Conclusion}

Poor nutritional status confers an increased risk of major postoperative complications and being discharged to a facility in non-upper gastrointestinal cancer patients. There was a correlation between the number of malnutrition parameters and the risk of complications.

Keywords: malnutrition; nutritional status; outcome assessment; surgical oncology

This article is protected by copyright. All rights reserved. 


\section{Introduction}

It is estimated that approximately $31 \%$ of all individuals admitted to a hospital for any cause are malnourished, with cancer patients experiencing even higher rates due to the systemic nature of malignancy $(1,2)$. Malnutrition has a multitude of clinical implications having been demonstrated to adversely affect mortality, morbidity and length of hospital stay amongst heterogeneous populations of surgical patients(3). However, there is considerable disparity in the assessment of malnutrition. Formal assessments such as the Nutritional Risk Screening and Malnutrition Universal Screening Tool have been utilized by a number of studies to measure nutritional status but they are infrequently employed in the clinical setting(4). Similarly, although sarcopenia has been associated with poor nutritional status, its assessment in routine practice is complicated(5). It is more common for patient characteristics such as low BMI, hypoalbuminemia and a loss of weight to form the basis of clinical assessment(6). In fact, these three parameters are endorsed as the metrics for perioperative nutritional assessment in geriatric patients by the American Geriatric Society best practice guidelines(7). However, there have been conflicting reports regarding the association, or lack thereof, between these factors and surgical outcomes $(8,9)$. Therefore, their utility in clinical practice as an indicator of malnutrition is debated. Furthermore, to the authors' knowledge, no study has evaluated these parameters in the context of surgical outcomes of 
patients with abdominopelvic cancers. Accurate nutritional assessment is of particular importance in major oncological cases where there is a relatively high risk of complications due to the complexity of the procedure and thus optimizing nutritional status has the potential to improve outcomes.

This study aimed to assess the prevalence of malnutrition as determined by the presence or absence of one or more of the three clinical parameters amongst patients undergoing major cancer surgery and its impact on outcomes.

\section{Materials and Methods}

\section{Database and Patients}

The American College of Surgeons National Surgical Quality Improvement Program (ACS-NSQIP) database is a prospective, multi-institutional program that records healthcare information for a random sample of surgical procedures at each site. Over 600 centers in the United States contribute information to the program. In addition to preoperative data, post-operative outcomes for up to 30 days following surgery are captured in the database. Clinical reviewers who undergo extensive modular training and annual certification obtain information directly from clinical records input data at each site. The recorded data is audited and has been shown to be highly reliable with only a $2.3 \%$ disagreement rate(10).

This article is protected by copyright. All rights reserved. 
The ACS-NSQIP Participant Data Use File from 2005 to 2016 was queried for elective cases of colectomy, cystectomy, esophagectomy, gastrectomy, hysterectomy, nephrectomy, pancreatectomy, pneumonectomy and prostatectomy using Current Procedural Terminology (CPT) codes (Appendix 1). The reason for choosing these procedures is that they have been adopted in other large studies examining major cancer surgery and, a subset of these procedures has been commonly used by the LeapFrog group (an initiative aiming to improve care and patient decision-making by publically reporting critical safety and quality information) as procedures to ascertain quality outcomes at the hospital level. These procedures were specifically chosen because the outcomes of these high-risk procedures have been strongly associated with quality of care(12). Only those procedures with a concomitant diagnosis of cancer based on International Classification of Diseases coding were included in the study (Appendix 1). Cases with metastatic disease were excluded from this study.

Patients were stratified into groups based on the presence of a combination of malnutrition markers: $\mathrm{BMI}<18.5 \mathrm{~kg} / \mathrm{m}^{2}$, preoperative serum albumin $<3.0 \mathrm{~g} / \mathrm{dL}$ or more than $10 \%$ weight loss in the last 6 months. These metrics are endorsed by The European Society for Clinical Nutrition and Metabolism as ideal for assessment of malnutrition(13).

This article is protected by copyright. All rights reserved. 


\section{Outcomes}

Postoperative complications are recorded in NSQIP and graded according to the Clavien-Dindo classification system. The correlation between each outcome recorded in the database and Clavien grade has been described previously(14) (Appendix 2). The primary endpoint was the incidence of a major Clavien complications defined as grade III-V. Discharge to a facility was evaluated as a secondary endpoint.

\section{Covariates}

Demographic variables collected include age, gender and race. Other pre-operative variables that were recorded in the dataset and uniformly coded throughout 20072016 were included.

\section{Statistical analysis}

Indicators of malnutrition (BMI, serum albumin and weight loss) were re-coded as dichotomous variables according to parameters described above to denote the poor nutritional status. Propensity score analysis was performed to address differences in baseline characteristics between individuals that are malnourished and those that are not. The propensity score was developed by including all pre-operative patient characteristics and procedure type. Matching was performed using the nearest 
neighbour technique in a 2:1 ratio. The balance of baseline characteristics between the groups was assessed both numerically and graphically. The difference in Clavien III-V complications between the two groups were compared between those meeting the definition of being malnourished and those who do not. Subgroup analysis, including repeating the matching process, was performed on upper gastrointestinal cases that have been described to potentiate malnutrition (esophagectomy, gastrectomy and pancreatectomy) compared to the remaining procedures(3).

To test whether the number of parameters correlated to severity of malnutrition, the incidence of complications between individuals that possessed none to all three malnutrition parameters were compared. Each combination of parameters was also compared to further distinguish risk.

Continuous variables are reported as means or medians if not normally distributed and compared with $\mathrm{t}$ tests. Categorical variables are reported as counts and percentages and compared using $\chi^{2}$ tests. Multivariable regression analyses were performed for the endpoints of interest. All $p$ values were two-sided and the level of significance set at 0.05 . Analysis was performed using performed in R ( $R$ Foundation for Statistical Computing, Vienna, Austria) version 3.4.

\section{Results}

This article is protected by copyright. All rights reserved. 
The cohort selection is outlined in Appendix 3. Missing data on one or more of the malnutrition parameters was present in 19,008 cases of which albumin was the most common (99.7\%, $n=18,946)$. Demographic characteristics for the 31,207 included cases are outlined in Appendix 4.

\section{Prevalence of malnutrition}

Overall, $8.5 \%(n=2,665)$ had at least one indicator of malnutrition. The prevalence of malnutrition for colectomy, cystectomy, esophagectomy, gastrectomy, hysterectomy, nephrectomy, pancreatectomy, pneumonectomy and prostatectomy was $8.7 \%, 5.4 \%, 28.1 \%, 15.0 \%, 2.4 \%, 4.9 \%, 26.2 \%, 7.2 \%$ and $0.6 \%$, respectively. Greater than $10 \%$ weight loss only in the preceding six months prior to surgery was the most common malnutrition indicator experienced by $4.9 \%(n=1,499)$ of individuals followed by hypoalbuminemia in $3.2 \%(n=1,008)$ and low BMI in $1.5 \%$ $(n=474)$.

\section{Major complications}

The overall incidence of Clavien III-V complications across all procedures was $5.8 \%$ $(n=1,825)$. There was a significant association between the prevalence of malnutrition by procedure and the incidence of Clavien III-V complication $(p=0.0113)$ (Figure 1). There was no difference in baseline covariates and propensity

This article is protected by copyright. All rights reserved. 
scores after the matching process (Appendix 5). Cases classified as malnourished had a higher incidence of Clavien III-V complications compared to controls ( $11.3 \%$ vs 9.6\%, $\mathrm{p}=0.018)$. This corresponded to an odds ratio (OR) of 1.34 [95\% Cl 1.16-1.55]. Malnutrition was only significantly associated with non-upper gastrointestinal cases (9.8\% vs 5.9\%, $p<0.001 ;$ OR 1.73 [95\%Cl 1.35-2.20] but not upper-gastrointestinal cases $(12.9 \%$ vs $13.1 \%, p=0.85 ;$ OR 1.02 [95\% Cl 0.82-1.23].

There was a significant association between the number of malnourished parameters met and the incidence of Clavien III-V complications (Figure 2). Amongst the unmatched cohort, the adjusted OR for experiencing a complication when possessing one, two or three malnutrition parameters compared to none was 1.90 [95\%Cl 1.65-2.19], 2.31 [95\%C 1.61-3.32] and 5.47 [95\% Cl 1.85-16.17], respectively $(p<0.001)$. The multivariable results for each combination of parameters is outlined in Table 1.

\section{Secondary outcomes}

The incidence of being discharged to a facility in cases with no, one, two or three malnutrition parameters was $5.7 \%, 14.6 \%, 16.7 \%$ and $29.4 \%$, respectively $(p<0.001)$. The multivariable results for each combination of parameters is outlined in Table 1.

This article is protected by copyright. All rights reserved. 
There was a higher incidence of being discharged to a facility amongst those malnourished compared to those that had a satisfactory nutritional status ( $15.0 \%$ vs $11.6 \%, p<0.001)$. The corresponding OR was $1.34[95 \% \mathrm{Cl}, 1.16-1.55]$. Similar to the relationship observed with major Clavien complications, malnutrition was not associated with rates of discharge to a facility amongst upper gastrointestinal cases (16.1\% vs $14.4 \%, \mathrm{p}=0.19 ;$ OR 1.14 [95\% $\mathrm{Cl} 0.94-1.39]$ ) but did show a relationship in the remaining cases (14.1\% vs 9.6\%, $\mathrm{p}<0.01$; OR $1.54[95 \% \mathrm{Cl} 1.25-1.88])$.

\section{Discussion}

The findings from this study support the association between malnutrition and adverse surgical outcomes in non-upper gastrointestinal cancer cases(3). Given that nearly one in ten patients possessed at least one marker of poor nutrition, it suggests that malnutrition is a considerable issue amongst patients undergoing major cancer surgery. It should be recognized that this could be an underestimate as the weighted average of the prevalence of malnutrition in US and European studies has been reported to be $31.4 \%(1)$. Using similar parameters to define nutritional status, albeit with a more lenient definition of hypoalbuminemia, Gregg and colleagues reported a prevalence of $19 \%$ amongst patients undergoing radical cystectomy for bladder cancer(15). Additionally, cancer patients have been observed

This article is protected by copyright. All rights reserved. 
to have higher rates of poor nutrition(16). However, considering cases in our study were deemed to be satisfactorily fit for major cancer surgery, they may represent a 'healthier' subgroup of all cancer patients, which may explain the discrepancy in malnutrition prevalence rates. Furthermore, the difference in the reported burden of malnutrition may also arise from the different definitions and assessments used(17). Nonetheless, it is evident that poor nutrition encumbers a considerable proportion of patients undergoing major cancer surgery and therefore is a factor that should be screened for.

Hypoalbuminaemia has been recognized as a predictor of operative morbidity and mortality in several trials $(23,24)$. The results from our study supported this with low serum albumin significantly increasing the likelihood of all adverse endpoints. However, it should be noted that there are some studies that have found no association between hypoalbuminaemia and surgical outcomes(25). This could be explained by the multitude of factors that can influence serum albumin levels and the suggested lack of association to nutritional status(26). The phenomenon of 'sarcopenic obesity' where an increase in fat mass occurs concurrently with a loss of skeletal muscle could explain the discrepancy between serum albumin levels and nutritional status(5). Thus, the association observed between low albumin and

This article is protected by copyright. All rights reserved. 
adverse outcomes could be driven by factors other than malnutrition. Furthermore, there is also a co-existing systemic inflammatory process seen in cancer patients may be playing a role in the observed discordance in nutritional parameters and outcomes(27). It is reported that up to $50 \%$ of patients have an acute-phase protein response, including increased albumin, at the time of upper-gastrointestinal cancer diagnosis(28). Hence, even patients with adequate nutritional status could have a low serum albumin which could confound the results.

The discrepant results with hypoalbuminaemia and the potential for confounders highlights the limitations of completely relying on clinical parameters to assess malnutrition. The subjective global assessment of nutritional status is a validated, reliable tool that can identify patients at nutritional risk and has been shown to be predictive of complications in patients undergoing major abdominal surgery(30). However, it is infrequently used during the pre-operative patient work-up due to inadequate education of clinicians and the time required, compared to laboratory markers. Similarly, anthropometric measurements and hand grip strength may be better able to characterise nutritional status by assessing changes in muscle mass and subcutaneous fat but require specialist equipment and assessment(31). As a result, the parameters used in our study are often relied upon because they are

This article is protected by copyright. All rights reserved. 
accessible and do not add extra time burden. Nonetheless, improved education of clinicians and pre-operative dietician assessment may facilitate more accurate determination of nutritional status.

This study has a number of limitations. It is primarily limited by shortcomings of the dataset, which only recorded postoperative outcomes up to 30 days following surgery. We are also reliant on the accuracy of the database but NSQIP uses highlytrained clinical reviewers and conducts regular audits to ensure data reliability. There may be some degree of selection bias impacting the results of our study as a considerable number of patients were excluded due to a missing nutritional measure and these patients had different clinical characteristics (younger, had a greater mean BMI, a lower mean NSQIP morbidity risk score, tended to be male, have a lower ASA and a greater proportion undergoing prostatectomy, hysterectomy and nephrectomy) and a lower occurrence of complications (3.9\% vs 5.9\%, $p<0.001$ ). Furthermore, variables not recorded in the dataset but could have influenced outcomes were unable to be accounted for, leading to unmeasured confounding (e.g. surgical approach). It could also be suggested that exclusion of patients due to missing values for nutritional parameters (primarily serum albumin) may underestimate effect size because healthier patients who likely have a normal serum albumin value are less likely to have this checked pre-operatively. The results may also not be generalizable to institutions not participating in NSQIP as they may be

This article is protected by copyright. All rights reserved. 
intrinsically different to NSQIP-affiliated centres. Finally, this study is unable to clearly determine whether the evaluated parameters reflect true malnutrition or is related to the underlying disease process, which is an area that requires further research.

Considering that resection generally needs to occur in a timely manner to provide the best chance of oncological success, it may not be feasible to optimize nutritional status pre-operatively in all patients classified as 'malnourished'. However, by taking into regard a combination of parameters, it may be possible to identify a sub-group of patients most at risk of adverse post-operative events - for example, those who have all markers of malnutrition (Figure 2) - and in whom the benefit of improving nutritional status outweighs the risks of delaying surgery. Jie and colleagues reported that abdominal surgical patients at severe nutritional risk who receive preoperative nutritional support had a lower rate of complications ( $25.6 \%$ vs $50.6 \%, p=0.008$ ) and shorter length of stay than those that did not have their nutritional status optimized(21). There was no observed effect of nutritional support in patients with lower severity of malnutrition in this study. Delaying resection in severe nutritional risk patients is supported by the ESPEN guidelines, however their definition of 'severe' includes any individual with at least one of the poor nutrition markers used in this study(22).

This article is protected by copyright. All rights reserved. 


\section{Conclusion}

Malnutrition burdens a considerable proportion of patients undergoing major cancer surgery. Patients classified as being malnourished had a greater likelihood of experiencing a major postoperative complication, having an extended duration of admission and being discharged to a facility. Furthermore, clinicians can be better informed by considering serum albumin, recent weight loss and BMI together rather than individually when assessing nutritional status. Being able to predict discharge destination may allow one to facilitate discharge planning at an earlier time point.

This article is protected by copyright. All rights reserved. 


\section{References}

1. Norman K, Pichard C, Lochs H, Pirlich M. Prognostic impact of disease-related malnutrition. Clinical Nutrition. 2008;27(1):5-15.

2. Fearon KC, Voss AC, Hustead DS. Definition of cancer cachexia: effect of weight loss, reduced food intake, and systemic inflammation on functional status and prognosis. The American journal of clinical nutrition. 2006;83(6):1345-50.

3. Leandro-Merhi VA, de Aquino JLB. Determinants of Malnutrition and Postoperative Complications in Hospitalized Surgical Patients. Journal of Health, Population, and Nutrition. 2014;32(3):400-10.

4. Patel V, Romano M, Corkins MR, DiMaria-Ghalili RA, Earthman C, Malone A, et al. Nutrition Screening and Assessment in Hospitalized Patients: A Survey of Current Practice in the United States. Nutrition in clinical practice : official publication of the American Society for Parenteral and Enteral Nutrition. 2014;29(4):483-90.

5. Santilli V, Bernetti A, Mangone M, Paoloni M. Clinical definition of sarcopenia. Clinical Cases in Mineral and Bone Metabolism. 2014;11(3):177-80.

6. Bharadwaj S, Ginoya S, Tandon P, Gohel TD, Guirguis J, Vallabh H, et al. Malnutrition: laboratory markers vs nutritional assessment. Gastroenterology Report. 2016;4(4):272-80.

7. Mohanty S, Rosenthal RA, Russell MM, Neuman MD, Ko CY, Esnaola NF. Optimal perioperative management of the geriatric patient: a best practices guideline from the American College of Surgeons NSQIP and the American Geriatrics Society. Journal of the American College of Surgeons. 2016;222(5):930-47.

8. Pacelli F, Bossola M, Rosa F, Tortorelli AP, Papa V, Doglietto GB. Is malnutrition still a risk factor of postoperative complications in gastric cancer surgery? Clinical nutrition (Edinburgh, Scotland). 2008;27(3):398-407.

9. Hu W-H, Cajas-Monson LC, Eisenstein S, Parry L, Cosman B, Ramamoorthy S. Preoperative malnutrition assessments as predictors of postoperative mortality and morbidity in colorectal cancer: an analysis of ACS-NSQIP. Nutrition Journal. 2015;14(1):91.

10. American College of Surgeons National Surgical Quality Improvement Program. User guide for the 2016 participant use data file 2016 [Available from: https://www.facs.org/ /media/files/quality\%20programs/nsqip/nsqip puf userguid e 2016.ashx.

11. Goodney PP, Stukel TA, Lucas FL, Finlayson EV, Birkmeyer JD. Hospital volume, length of stay, and readmission rates in high-risk surgery. Ann Surg. 2003;238(2):161-7.

12. Birkmeyer JD, Stukel TA, Siewers AE, Goodney PP, Wennberg DE, Lucas FL. Surgeon Volume and Operative Mortality in the United States. New England Journal of Medicine. 2003;349(22):2117-27. 
13. Weimann A, Braga $M$, Harsanyi L, Laviano A, Ljungqvist $O$, Soeters $P$, et al. ESPEN Guidelines on Enteral Nutrition: Surgery including Organ Transplantation. Clinical Nutrition. 2006;25(2):224-44.

14. Chimukangara M, Frelich MJ, Bosler ME, Rein LE, Szabo A, Gould JC. The impact of frailty on outcomes of paraesophageal hernia repair. The Journal of surgical research. 2016;202(2):259-66.

15. Gregg JR, Cookson MS, Phillips S, Salem S, Chang SS, Clark PE, et al. Effect of preoperative nutritional deficiency on mortality after radical cystectomy for bladder cancer. The Journal of urology. 2011;185(1):90-6.

16. Gyan E, Raynard B, Durand JP, Lacau Saint Guily J, Gouy S, Movschin ML, et al. Malnutrition in Patients With Cancer. JPEN Journal of parenteral and enteral nutrition. 2017:148607116688881.

17. Cerantola Y, Valerio M, Hubner M, Iglesias K, Vaucher L, Jichlinski P. Are Patients at Nutritional Risk More Prone to Complications after Major Urological Surgery? The Journal of urology. 2013;190(6):2126-32.

18. Garth AK, Newsome CM, Simmance N, Crowe TC. Nutritional status, nutrition practices and post-operative complications in patients with gastrointestinal cancer. Journal of human nutrition and dietetics : the official journal of the British Dietetic Association. 2010;23(4):393-401.

19. Schiesser M, Kirchhoff P, Müller MK, Schäfer M, Clavien P-A. The correlation of nutrition risk index, nutrition risk score, and bioimpedance analysis with postoperative complications in patients undergoing gastrointestinal surgery. Surgery. 2009;145(5):519-26.

20. Snider JT, Linthicum MT, Wu Y, LaVallee C, Lakdawalla DN, Hegazi R, et al. Economic burden of community-based disease-associated malnutrition in the United States. JPEN Journal of parenteral and enteral nutrition. 2014;38(2 Suppl):77s-85s. 21. Jie B, Jiang Z-M, Nolan MT, Zhu S-N, Yu K, Kondrup J. Impact of preoperative nutritional support on clinical outcome in abdominal surgical patients at nutritional risk. Nutrition. 2012;28(10):1022-7.

22. Weimann A, Braga M, Carli F, Higashiguchi T, Hübner M, Klek S, et al. ESPEN guideline: Clinical nutrition in surgery. Clinical Nutrition. 2017;36(3):623-50.

23. Gibbs J, Cull W, Henderson W, Daley J, Hur K, Khuri SF. Preoperative serum albumin level as a predictor of operative mortality and morbidity: Results from the national va surgical risk study. Archives of Surgery. 1999;134(1):36-42.

24. Badia-Tahull MB, Llop-Talaveron J, Fort-Casamartina E, Farran-Teixidor L, Ramon-Torrel JM, Jódar-Masanés R. Preoperative albumin as a predictor of outcome in gastrointestinal surgery. e-SPEN, the European e-Journal of Clinical Nutrition and Metabolism. 2009;4(5):e248-e51.

25. Lim S, Kim BD, Kim JY, Ver Halen JP. Preoperative Albumin Alone is Not a Predictor of 30-Day Outcomes in Pressure Ulcer Patients: A Matched Propensity- 
Score Analysis of the 2006-2011 NSQIP Datasets. Annals of plastic surgery. 2015;75(4):439-47.

26. Covinsky KE, Covinsky MH, Palmer RM, Sehgal AR. Serum albumin concentration and clinical assessments of nutritional status in hospitalized older people: different sides of different coins? Journal of the American Geriatrics Society. 2002;50(4):631-7.

27. Gangadharan A, Choi SE, Hassan A, Ayoub NM, Durante G, Balwani S, et al. Protein calorie malnutrition, nutritional intervention and personalized cancer care. Oncotarget. 2017;8(14):24009-30.

28. Gruys E, Toussaint MJM, Niewold TA, Koopmans SJ. Acute phase reaction and acute phase proteins. Journal of Zhejiang University Science B. 2005;6(11):1045-56.

29. Fukuda Y, Yamamoto K, Hirao M, Nishikawa K, Maeda S, Haraguchi N, et al. Prevalence of Malnutrition Among Gastric Cancer Patients Undergoing Gastrectomy and Optimal Preoperative Nutritional Support for Preventing Surgical Site Infections. Annals of surgical oncology. 2015;22 Suppl 3:S778-85.

30. Sungurtekin H, Sungurtekin U, Balci C, Zencir M, Erdem E. The influence of nutritional status on complications after major intraabdominal surgery. Journal of the American College of Nutrition. 2004;23(3):227-32.

31. Windsor JA, Hill GL. Grip strength: a measure of the proportion of protein loss in surgical patients. The British journal of surgery. 1988;75(9):880-2.

32. Drover JW, Dhaliwal R, Weitzel L, Wischmeyer PE, Ochoa JB, Heyland DK. Perioperative use of arginine-supplemented diets: a systematic review of the evidence. Journal of the American College of Surgeons. 2011;212(3):385-99, 99.e1. 
Figure 1. Association between the prevalence of malnutrition and complication rate $(p=0.0113)$

Legend: blue line represents linear regression line and surrounding shading represents the $95 \%$ confidence interval

Figure 2. Incidence of Clavien III-V complication by the number of malnourished parameters $(p<0.001)$

This article is protected by copyright. All rights reserved. 
Table 1. Multivariable logistic regression results for each combination of malnutrition parameters

\begin{tabular}{|l|l|l|l|l|}
\hline & \multicolumn{2}{|l|}{ Clavien III-V complications } & \multicolumn{2}{l|}{ Discharge to a facility } \\
\hline Nutrition Status & OR & $95 \% \mathrm{Cl}$ & OR & $95 \% \mathrm{Cl}$ \\
\hline Normal & 1.00 & & 1.00 & \\
\hline Low albumin only & 2.05 & $1.64-2.56$ & 2.39 & $1.94-2.96$ \\
\hline Low BMI only & 1.54 & $1.02-2.31$ & 1.55 & $1.06-2.27$ \\
\hline Weight loss only & 1.89 & $1.56-2.28$ & 1.93 & $1.60-2.35$ \\
\hline Low BMI \& weight loss & 2.69 & $1.49-4.84$ & 3.07 & $1.71-5.49$ \\
\hline $\begin{array}{l}\text { Low albumin \& weight } \\
\text { loss }\end{array}$ & 2.33 & $1.46-3.71$ & 1.86 & $1.12-3.11$ \\
\hline Low albumin \& BMI & 0.72 & $0.09-5.54$ & 2.66 & $0.91-7.84$ \\
\hline $\begin{array}{l}\text { Low albumin, BMI \& } \\
\text { weight loss }\end{array}$ & 5.47 & $1.85-16.16$ & 2.71 & $0.79-9.31$ \\
\hline
\end{tabular}

This article is protected by copyright. All rights reserved. 


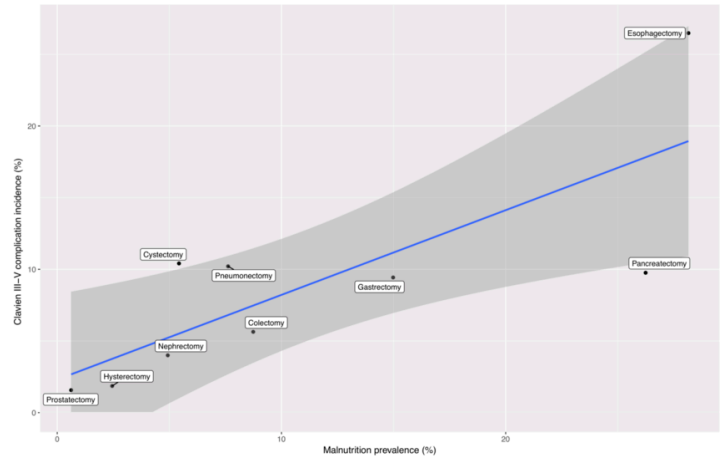

ANS_15129_Figure 1_fixed.tiff

This article is protected by copyright. All rights reserved. 


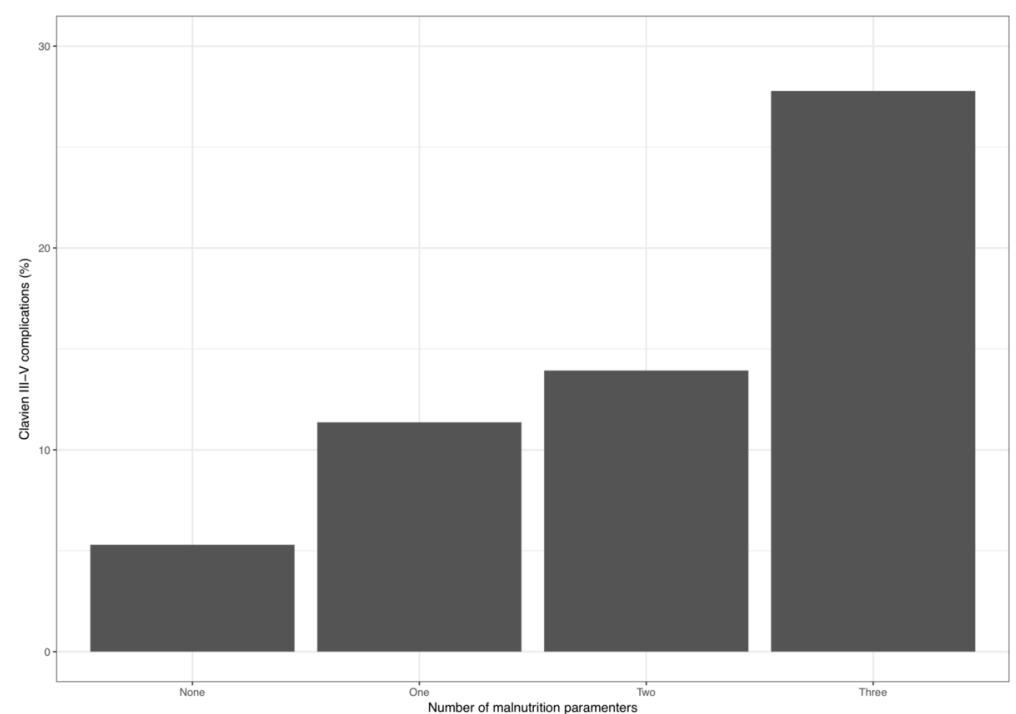

ANS_15129_Figure_2_malnutrition.tiff

This article is protected by copyright. All rights reserved. 


\section{University Library}

\section{- M M N E R VA A gateway to Melbourne's research publications}

Minerva Access is the Institutional Repository of The University of Melbourne

Author/s:

Sathianathen, NJ;Kwaan, M;Lawrentschuk, N;Weight, CJ;Kim, SP;Murphy, DG;Moon, DA; Konety, BR

Title:

Adverse impact of malnutrition markers on major abdominopelvic cancer surgery

Date:

2019-05-01

Citation:

Sathianathen, N. J., Kwaan, M., Lawrentschuk, N., Weight, C. J., Kim, S. P., Murphy, D. G., Moon, D. A. \& Konety, B. R. (2019). Adverse impact of malnutrition markers on major abdominopelvic cancer surgery. ANZ JOURNAL OF SURGERY, 89 (5), pp.509-514. https:// doi.org/10.1111/ans.15129.

Persistent Link:

http://hdl.handle.net/11343/285685 